\title{
Características de la producción petrolera a partir de los años 70: Un análisis del caso argentino en el esquema de acumulación mundial
}

Jimena Segura*

Resumen: Bajo el modo de producción capitalista, el precio de las mercancías se convierte en la principal señal para el capital en su movimiento entre las distintas esferas productivas. A partir del año 2003, el precio del petróleo presentó una suba histórica comparable a la de fines de la década del '70. Sin embargo, a diferencia de esos años, el aumento del precio no resultó en aumentos significativos en la capacidad productiva del sector. Es objetivo de este trabajo dar cuenta de las determinaciones de la inversión de capital en el sector energético que explique las características específicas en la producción actual, en donde inevitablemente se toma en cuenta como factor a considerar el ingreso progresivo del capital financiero en torno a la producción del sector. Siendo históricamente la Argentina poseedora de un gran potencial en materia de energía, queda por resolver las cuestiones que llevan a que la situación energética local, sea presa de un debate que apunte a señalar las posibilidades de una crisis en un mediano o corto plazo, al enfrentar ciertas deficiencias en casi todos los aspectos que hacen al abastecimiento de energía.

Palabras clave: petróleo-inversión-Argentina

\begin{abstract}
Under the capitalist mode of production, the price of the goods becomes the main signal for capital in their movement between different productive areas. As of 2003, the oil price presented a comparable rise to that of the end of the 70's. However unlike those years, the increase in the price did not result in significant increases in the productive capacity of the sector. It is a goal of this work to account for the determinations of capital investment in the energy sector that explained the specifi characteristics in this type of production, where inevitably is considered as a main factor the progressive incidence of financial capital. Historically Argentina had a great potential in the field of energy, what lead us to question the issues that present the local energy situation as a posible crisis in the short or medium term, at facing certain shortcomings in almost all aspects that make the supply of energy.
\end{abstract}

Key words: oil-investement-Argentina

\footnotetext{
* Licenciada em Economia. Doutoranda na Faculdade de Ciências Econômicas UBA. Docente da Universidade de Buenos Aires. jimesegu@hotmail.com
}

Latitude, vol. 3, n² 2, pp. 41-64, 2009

DOI: https://doi.org/10.28998/2179-5428.20090203 


\section{Precios locales e internacionales}

La reestructuración de la industria petrolera argentina, a partir de las reformas de los '90, las cuales tuvieron como eje la privatización de Yacimientos Petrolíferos Fiscales, concretaron el cambio de política nacional, respecto a la producción energética. Uno de sus principales objetivos fue el realineamiento de los precios locales respecto a los internacionales a través del Plan Argentina de 1991. Existía previamente una política local de precios del petróleo fijados mediante acuerdos entre YPF y el sector privado que, en tanto se mantenían por debajo de los precios internacionales, dando lugar a una apropiación de la renta petrolera por parte del capital local consumidor de este insumo energético básico. Con la reforma, en primer lugar, se transfirieron áreas de exploración y producción al sector privado, se otorgó la libre disponibilidad del crudo obtenido, la desregulación de los precios y la eliminación de trabas al comercio exterior ${ }^{1}$. Posteriormente, se vendieron las refinerías y otros activos de YPF y la cesión de sus porcentajes de asociación en otras empresas. En 1990 se había transformado a YPF en una sociedad anónima que se acercó gradualmente a su privatización, culminando hacia el año 1993. Los yacimientos estatales se convirtieron en licencias y concesiones de explotación y, a partir de 1992, se federalizó el dominio de los hidrocarburos. De esta manera, se descentralizaba la apropiación estatal de la renta. Con la privatización, una mayor porción de la renta petrolera se privatiza y destina al capital invertido directamente en el sector. En esta primera venta de activos, se estima que el $63,1 \%$ pertenecía a fondos privados extranjeros, que se componía de fondos de inversión de EEUU, seguido de otros actores institucionales del Reino Unido y Francia². En 1999, la mayor parte de las acciones son vendidas a la española Repsol. En este sentido, la privatización de YPF consistió en un traspaso de gran parte de la renta petrolera nacional a disposición de capitales extranjeros.

A través de estas modificaciones, los precios internos del crudo en dólares se mostraron más estrechamente relacionados con aquellos correspondientes a las vicisitudes de la demanda y oferta mundial de petróleo (CUADRO 1)3.

\footnotetext{
${ }^{1}$ Instituto Argentino de la Energía "General Mosconi", Análisis del mercado de combustibles líquidos en la República Argentina", abril 2000.

${ }^{2}$ Kozujl R., Balance de la privatización de la industria petrolera en Argentina y su impacto sobre las inversiones y la competencia en los mercados minoristas de combustibles, Cepal, julio 2002.

${ }^{3}$ Kozujl R., Crisis de la industria del gas natural en la Argentina, Cepal marzo 2005.
} 


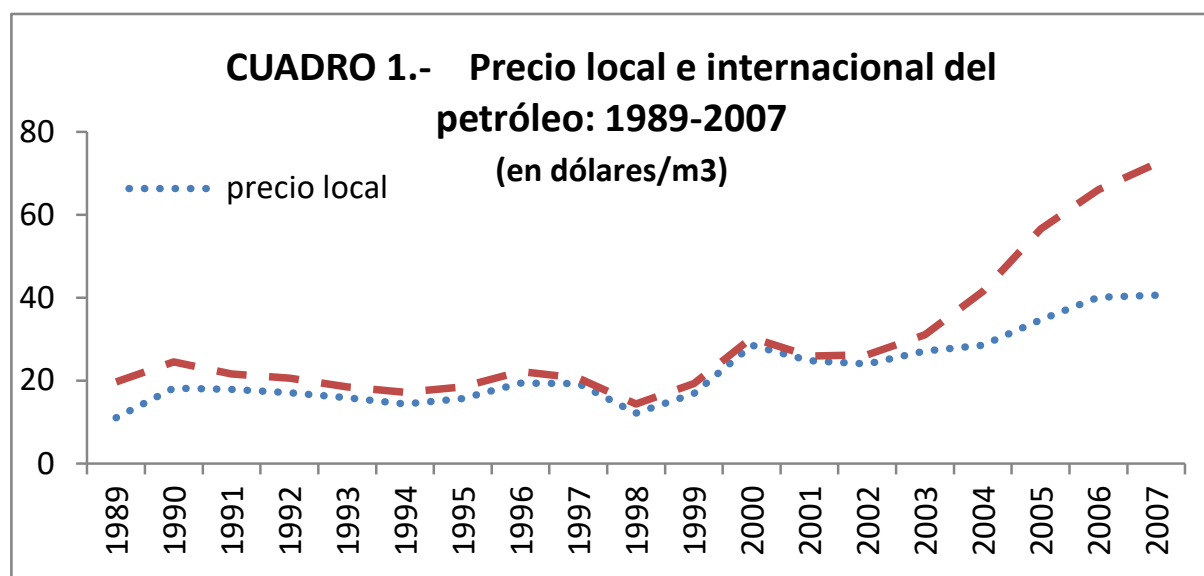

Fuente: precio local en Kozujl (marzo 2005) sobre la base de información de la Secretaría de Energía de la Nación y la Opep; precio internacional en Energy Information

A partir de la crisis petrolera de 1974, los precios locales habían quedado desfasados de los precios internacionales y, recién hacia 1988, comenzaron a equipararse, debido a la caída internacional de los precios ${ }^{4}$. Aún así, los valores se fueron ajustando y acompañando la histórica suba de la década del '70 (CUADRO 2)

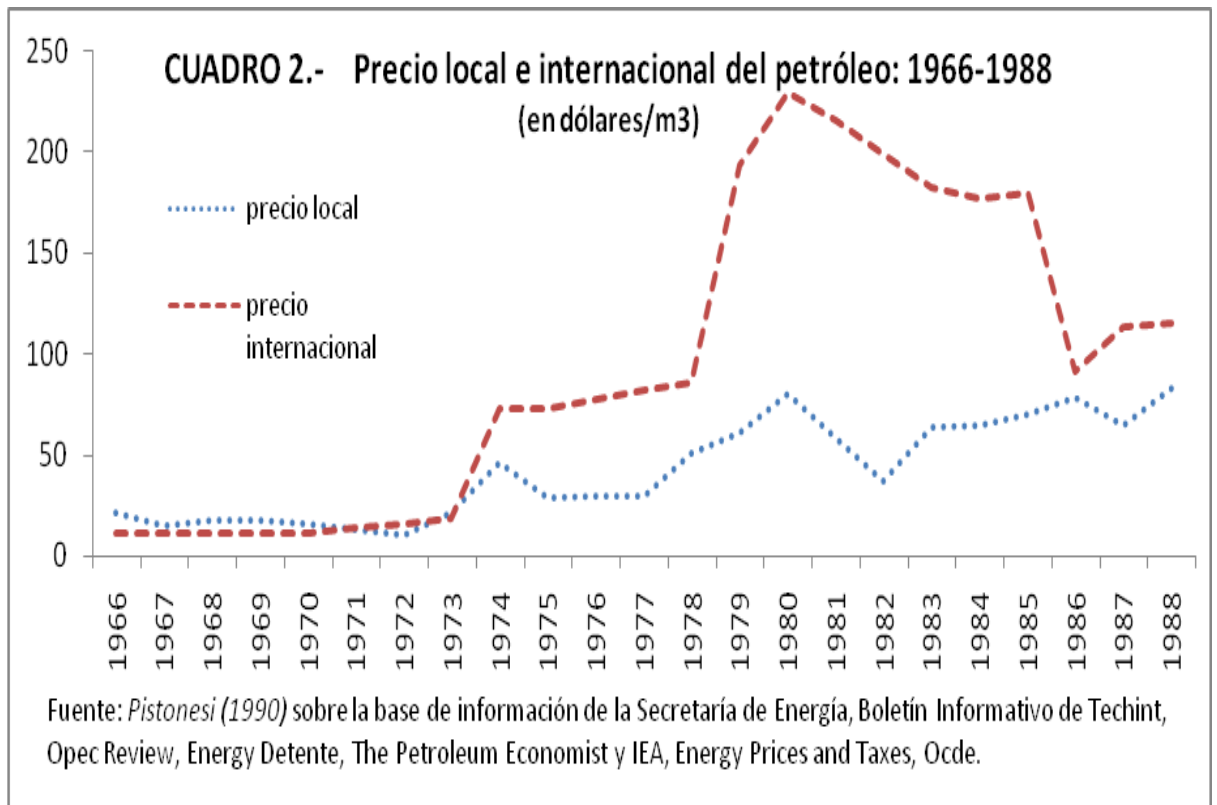

${ }^{4}$ Pistonesi, Figueroa de la Vega y Torres, Política de precios de la energía en la Argentina 1970-1989, Fundación Bariloche 1990.

Latitude, vol. 3, n², pp. 41-64, 2009. 
Características de la producción petrolera a partir de los años 70:

Un análisis del caso argentino en el esquema de acumulación mundial

YPF concentraba antes de la reforma, aproximadamente las dos terceras partes de la capacidad de refinación, donde mayormente se procesaba todo el crudo disponible y se exportaban los derivados no demandados por el mercado local. A partir de 1990, se procesa más crudo en las refinerías del país pero, luego de la venta de las refinerías, en 1993, disminuyen los niveles de procesamiento de derivados y su exportación. Argentina no era rentable a nivel regional en la etapa de downstream, las refinerías mostraban falencias. Por tanto, el incremento en la producción de crudo de este período fue a parar en gran parte a su exportación, para ser procesado en refinerías del exterior, ya que si bien aumenta la actividad económica local desde 1991 y por ende la demanda de petróleo, crece a su vez -y en especial a partir de 1993- la participación de la exportación de crudo respecto a la producción total (de hecho, en 1993, cae la tasa de crecimiento del producto bruto interno a precios constantes). De esta manera, podría decirse que la exportación de crudo fue el estímulo principal al aumento de la producción del período luego de la privatización (CUADRO 3). Esto refleja el distanciamiento de la producción energética de las necesidades directas del mercado local frente a la del capital petrolero extranjero que opera a nivel regional o trasnacional. En este sentido, el posterior desarrollo tecnológico y de inversión estará íntimamente ligado al interés de estos capitales en particular. Sobre esta base, la alineación de los precios locales con los internacionales fue esencial, especialmente en un contexto internacional de oscilaciones de precio del petróleo crudo.

\section{CUADRO 3.- Producción y exportación de crudo (en miles de m3)}

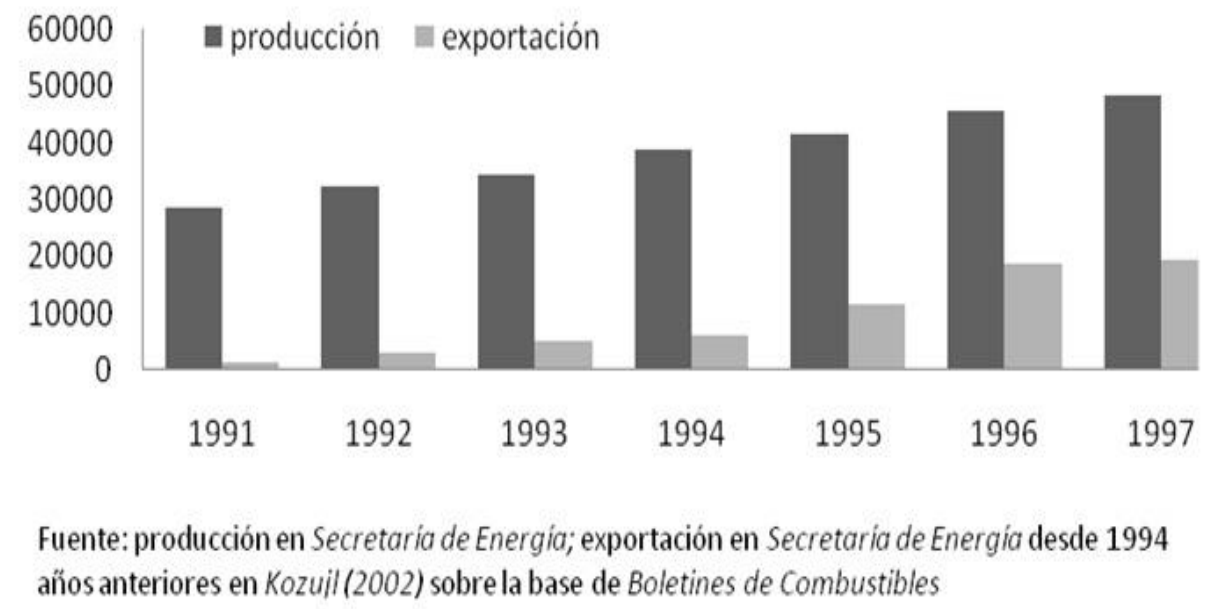




\section{Inversión y producción}

Posteriormente a 1993, año en que culmina el proceso de privatización de la producción, refinación y comercialización de crudo, se registra un aumento considerable en el número de pozos de petróleo terminados de explotación $(\mathrm{CUADRO} 4)^{5}$.

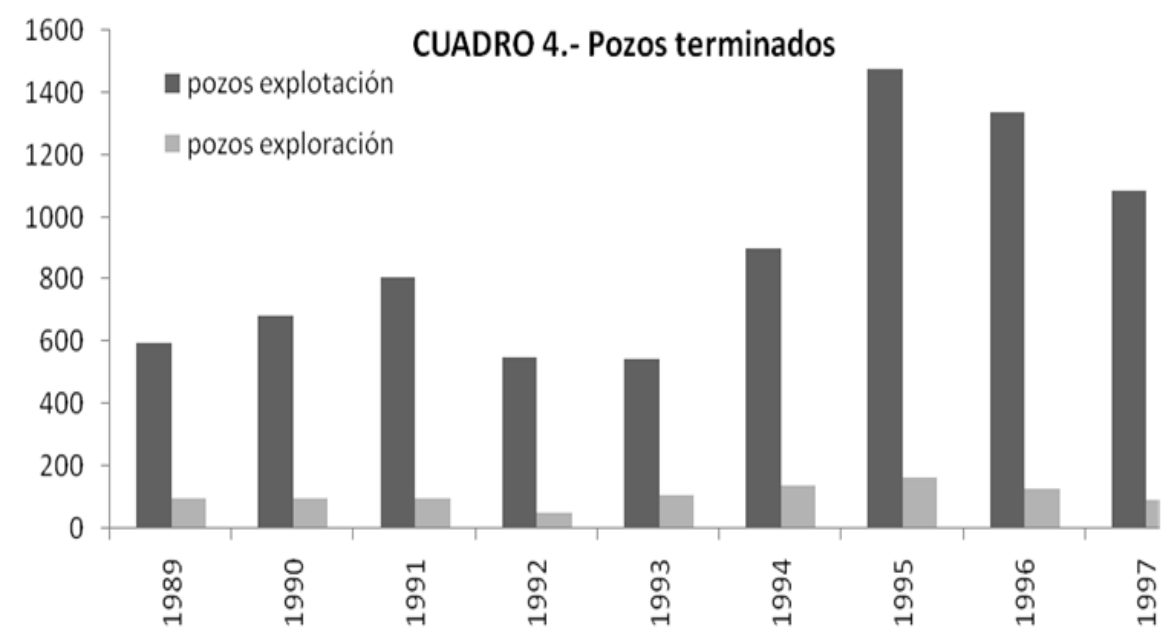

Fuente: en Gadano (1998) sobre la base de datos del Boletín de Combustibles.

A partir de 1996 y en mayor medida hacia 1997, el número de pozos terminados comienza a descender -cabe señalar que la tasa de éxito para 1996 y 1997 fue más alta que en los años previos ${ }^{6}$. En 1997 y 1998, los precios caen a partir de la crisis asiática que tuvo impacto mundial en la industria petrolera. Para estos años se reduce el número de pozos terminados en la Argentina y, como se verá posteriormente, la inversión en producción de petróleo a nivel mundial.

Aún así, si se observa el ciclo de producción de petróleo en Argentina durante la década de los ' 90 y se ve una tendencia ascendente que recién se revierte hacia 1999 (CUADRO 5).

${ }^{5}$ Gadano N., Determinantes de la inversión en el sector petróleo y gas de la Argentina, Cepal, octubre 1998.

${ }^{6}$ La tasa de éxito es la cantidad de pozos productivos respecto a los pozos de exploración. En 1997 fue del 59\%, en 1994, 1995 y 1996 fue de 41\%, 48\% y 53\% respectivamente (ver Gadano 1998). 


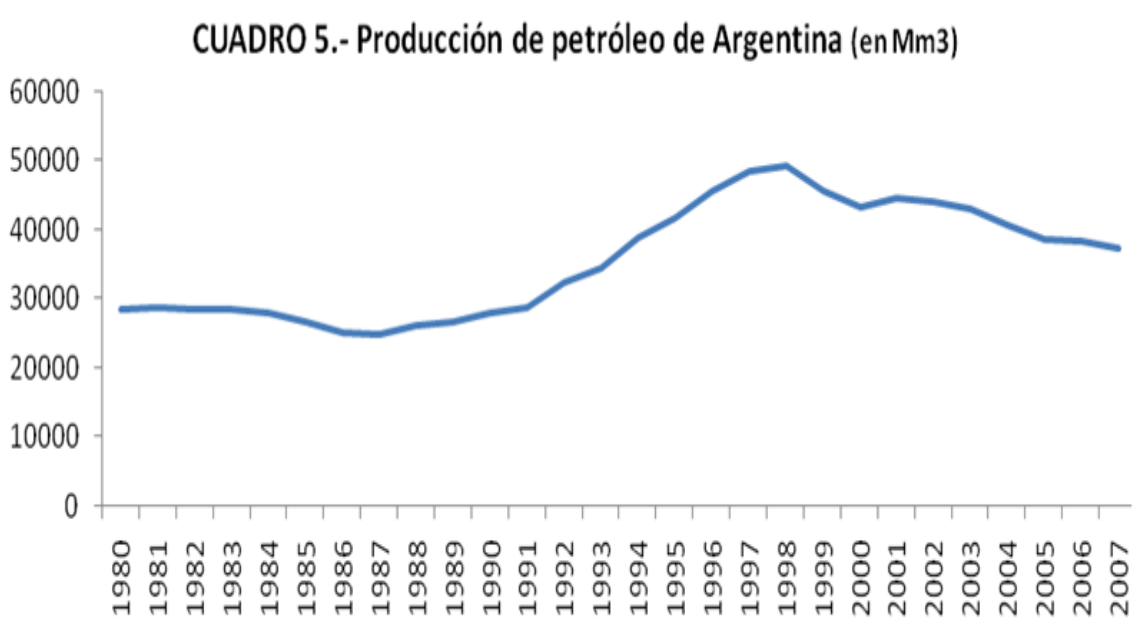

Fuente: Secretaria de Energia de la Nación

Este aumento en la producción nacional de petróleo, se basó en el crecimiento en la perforación de pozos de explotación, y del relativamente estancado número de pozos terminados de exploración, es decir, se trató de un aumento en la tasa de extracción sobre yacimientos ya existentes, facilitado por las innovaciones en la industria en recuperación secundaria ${ }^{7}$ (TABLA 1).

TABLA 1.-

\begin{tabular}{|c|c|c|c|}
\hline Período & $\begin{array}{c}\text { pozos } \\
\text { explotación } \\
\text { (promedio) }\end{array}$ & $\begin{array}{c}\text { pozos } \\
\text { exploración } \\
\text { (promedio) }\end{array}$ & $\begin{array}{c}\text { reservas/ } \\
\text { producción } \\
\text { (años) }\end{array}$ \\
\hline $1976-82$ & 583 & 105 & 14 \\
\hline $1983-89$ & 669 & 118 & 14 \\
\hline $1990-94$ & 698 & 99 & 10 \\
\hline $1995-00$ & 978 & 92 & 9 \\
\hline
\end{tabular}

Fuente: Kozujl (2002) sobre datos de la Secretaría de Energía e IAPG, Boletín de Combustibles varios

${ }^{7}$ La recuperación secundaria de petróleo es la obtenida por inyecciones de gas o agua en los pozos. 
El escenario de aumento de la producción de crudo y baja actividad exploratoria no es específico de Argentina y la política privatizadora local; por el contrario, y como veremos a continuación, esta relación aparentemente contradictoria se manifestó como un fenómeno de carácter mundial. En este sentido, podría entenderse las reformas de los '90 como la viabilizadora local de un proceso de carácter más general. Veamos de qué se trata.

\section{Transformaciones del mercado petrolero mundial}

En la década del '70, EEUU se queda sin capacidad propia de excedente de petróleo y se enlentece el ritmo de descubrimientos locales, por lo que el capital norteamericano reduce la inversión petrolera en su país. Siendo el principal consumidor mundial, aumenta la dependencia global sobre el petróleo de Medio Oriente, en tanto los descubrimientos de Alaska y del Mar del Norte aún no eran explotables por la falta de desarrollo tecnológico para la explotación en condiciones climáticas extremas y de gran profundidad off shore. A partir del golpe de estado de Gadaffi de 1969 en Libia, se refuerza el poder de los países petroleros, donde surgen campañas sobre la soberanía y el control de sus recursos energéticos. A lo largo de esta década, los países exportadores de petróleo aumentan su participación en los beneficios, y fijan precios más altos, sin consultar a las tradicionales compañías petroleras que producían el petróleo en sus países (generalmente asociadas en consorcios): Exxon, Mobil, Chevron, Texaco, Gulf, Royal Dutch Shell, British Petroleum, Jersey, Socony y Total. Por otra parte se sucede una ola de nacionalizaciones parciales de las filiales de estas empresas en Libia, Argelia, Irak y Kuwait. De esta forma, comienzan a proliferar nuevos tipos de contratos en que las grandes petroleras pasan de ser poseedoras de licencias y concesiones, a contratistas con derechos para explorar, producir y comercializar crudo.

El empeoramiento en las condiciones de oferta de petróleo de EEUU y el despunte de la inflación norteamericana, lleva a Nixon a abolir el sistema de cuotas de importación para morigerar el creciente precio del combustible, lo que dio lugar a un considerable aumento en las importaciones a través de las cuales las refinerías y petroleras que operaban en ese país compraban todo el petróleo al que lograran acceder. De esta forma, aumentan los precios de mercado respecto a los oficiales establecidos por contrato. Los países exportadores de petróleo, apuntaron a aumentar la porción obtenida del aumento del precio que básicamente acaparaban las empresas al comprar más barato el crudo de sus países y venderlo más caro en el exterior. El embargo de 1973, por el enfrentamiento árabe-israelí, fue el mecanismo para lograrlo. La apropiación de renta petrolera por parte de estos países había dado lugar al desarrollo de un capital local, que conjuntamente con el proceso de descolonialización y la crisis del capital norteamericano, transformaron gradualmente las características del mercado petrolero mundial. Las nuevas empresas estatales petroleras tenían la capacidad de imponer mayores condiciones a las empresas norteamericanas y europeas, en representación de los intereses de estos capitales locales más concentrados. Como forma de avance en la apropiación 
en el valor de sus recursos, mediante la nacionalización lograron, en muchos casos, dar fin a viejos canales de comercialización, en donde estas compañías revendían el crudo de Medio Oriente. Las empresas estatales ahora vendían directamente a muchos de sus consumidores; el petróleo vendido por los países directamente al mercado aumentó del 8\% en 1973 al $42 \%$ en $19799^{\circ}$.

En el período de posguerra, el petróleo ocupaba una porción cada vez más preponderante en el consumo total de energía. Las altas tasas de crecimiento mundial presionaban al aumento en la demanda mundial de crudo, dando lugar a un prominente mercado que se encontró con una nueva masa de productores de petróleo. Posteriormente al proceso de nacionalizaciones, la creación de numerosas empresas estatales y nuevos canales de comercio, fueron las condiciones que este acrecentado sector productivo ofreció para la conformación de nuevos nichos de ganancia para el capital. Por su parte, el proceso de desregulación financiera internacional de comienzos de los '70, la mayor movilidad de capital y el crecimiento del endeudamiento dieron lugar a la proliferación de inversores financieros, que encontraron en las características de este mercado una base para su acumulación.

A fines de 1978, los inversores compraban cada vez más petróleo frente al aumento de su precio. De esta manera, el sector se encontraba al límite de la capacidad productiva. Con la revolución iraní de 1978, se suspenden los acuerdos de compra-venta preestablecidos, por lo que aparecen una multitud de nuevos compradores por fuera de este tipo de contratos, entre los cuales los más dinámicos son Japón, refinerías independientes y algunas compañías estatales que acuden al mercado al contado para abastecerse. Se consuma, de esta manera, el fin del clásico sector integrado, al quebrarse los estrechos vínculos que históricamente existían entre el nivel inicial y final del ciclo productivo. Así, el mercado al contado y la intermediación pasan a ser el centro del negocio. Surgen una cantidad de nuevos intermediarios, como casas de comercio y especuladores, que obtienen ganancias en la compra y venta de crudo y en el arbitraje que ofrece las diferencias entre los precios al contado y los precios de contrato. Por otra parte, las nacionalizaciones habían llevado a las grandes compañías de petróleo a reorientar nuevamente sus inversiones. De esta manera, perdía la Opep su dominio mundial frente a las incorporaciones de Alaska, el Mar del Norte, México y otras regiones en el esquema mundial de producción. Crecía así también el número de productores en busca de nuevos mercados de consumo.

Durante los años de suba de precio del petróleo e inflación, el capital financiero requirió de un resguardo de valor que se orientó hacia las acciones petroleras y el financiamiento de inversiones destinadas a aquellas mercancías poseedoras de renta como el petróleo. Se invertía pidiendo prestado a bancos e inversores con petróleo de garantía, aumentó considerablemente el precio de los

${ }_{8}^{8}$ Yergin D., La historia del petróleo, Ed. Vergara 1992. 
terrenos dónde perforar y de las ciudades cercanas a los yacimientos, además se estimulaba a que gran parte del ahorro familiar se destinara al petróleo. La inminente crisis de sobreproducción ofrecía bajos rendimientos para otro tipo de instrumentos financieros. Por su parte, la creciente necesidad de capital para exploración y explotación de petróleo, especialmente en tanto se reorientaban las inversiones a pozos menos productivos encontró, en el desarrollo mundial del capital a crédito, la base para su desenvolvimiento.

Las altas tasas de crecimiento mundial de comienzo de la década, el alto precio del petróleo, el desarrollo del sector financiero -en parte estimulado por el incremento en la renta petrolera en países con capitales locales de un nivel de concentración en aumento pero aún limitado- encuentran su propio límite que se manifiesta por medio de la crisis de principios de los ' 80 . Las reservas mundiales de petróleo habían crecido enormemente, pero la demanda comenzaba a caer; la naciente década daba comienzo a un ciclo recesivo mundial y de descenso en el precio del crudo. A la consiguiente baja de precios colabora el hecho de que por los altos precios de los hidrocarburos, durante la década anterior, se había producido un incremento en la inversión de energías alternativas y en el aumento de la conservación o rendimiento energético.

El tráfico y comercio de crudo se había desarrollado dentro de las petroleras al nivel de establecerse en unidades o centros específicos, en donde aparecía el objetivo de la obtención de ganancia comercial, como finalidad propia y separada del ciclo productivo. Esta transformación al interior de las empresas está asociada al avance del poder del capital financiero sobre sus acciones. Por sus características, el capital a crédito, desligado de la relación directa con la producción, exige plazos cortos en su valorización que le dé la capacidad de rotar con mayor versatilidad de una esfera productiva a la otra. Si bien ello facilita la acumulación del capital total, le impone condiciones al ciclo productivo, modificando el carácter de la producción al interior de las unidades productivas. Las inversiones de largo plazo, y aquella considerada como la incorporación de medios de producción específicos vinculados a un sector productivo en particular, son cuestiones que encuentran poco eco en un período en que el capital se aferra a su significado más general: valor que busca valorizarse.

Los inversores institucionales (fondos de pensión, mutualistas y monetarios) poseían tres cuartas partes de las acciones de las grandes compañías petroleras, por lo que reclamaban mayores beneficios y en el corto plazo ${ }^{9}$. El valor de las acciones no reflejaba el de las reservas, ya que éstas no se valorizaban por la caída en la demanda y la consecuente y gradual desinversión en el sector. De esta manera, se avanzaba en la adquisición de reservas y activos para fusionar o quebrar capitales a fines de revalorizar sus acciones. Las inversiones se focalizaban en este tipo de operatorias que encontraban su base en la sobreproducción, la cual

${ }^{9}$ Id. Yergin 
hacía más barato comprar explotaciones existentes infravaloradas que añadir barriles por prospección. Por otra parte, estos inversores ya no estaban preocupados por la inflación y habían pasado del financiamiento de la producción de petróleo a este tipo de transacciones. En este marco, se avanza con la privatización de empresas o activos petroleros estatales, otorgando a esta forma de capital nuevas bases de extracción de ganancia en un período de deflación. En 1987 se privatiza la British Petroleum, la Kuwait Oil Company se asocia con refinerías privadas, Aramco de Arabia Saudita con Texaco forman una empresa mixta, en 1989 se privatiza Repsol ${ }^{10}$ y comienza un proceso de privatizaciones en América Latina. Este desarrollo en la centralización del sector se manifestó en la bancarrota de pequeños productores y recortes de personal al interior de las empresas. Y encuentra en la reducción de liquidez para financiar la prospección y explotación de petróleo, una de las bases concretas de su desenvolvimiento.

\section{Oscilaciones y guerra. Los años '90.}

Irak decide invadir Kuwait en agosto de 1990 dando así comienzo a la llamada Guerra del Golfo. Esta guerra tuvo como resultado acciones destructivas sobre pozos e infraestructura petrolera, lo que generó que en el mismo mes de la invasión, la producción de petróleo para los países de la OPEP cayera en un $14,3 \%{ }^{11}$. Este suceso implicó para el mes siguiente una escalada en el precio del petróleo que alcanzó los 33.6 dólares el barril12, o sea un aumento de más del $59 \%$ en un mes (ver CUADRO 1).

Existe en este período una particularidad a tener en cuenta. Al observar la década del '80, pueden encontrarse recortes de producción mensuales que no lograron la escalada del precio que se observó en 1990. A diferencia de ese entonces, en los '90 no se resurgía de un escenario de precios record, de la consecuente incorporación de nuevos capitales y de picos históricos en la producción. Por el contrario, la desinversión y desaparición de pozos menos productivos fue típico de esta industria en los años '80. Por ello, el progresivo aumento en el consumo y la producción desde fines de esta década, llevó a que esta contracción en la oferta repercutiera de forma inmediata en una suba del precio.

Sin embargo, la transitoriedad de este pico en el precio de principios de los '90 tiene que ver nuevamente con el nivel de acumulación de capital mundial para

${ }^{10}$ Entidades financieras como el Bilbao Bizcaya y la Caixa se apropian de la mayoría de sus acciones. En el año 2005 se retira BBVV y SCyV se apropia del 20\% de las acciones de Repsol. SCyV es una entidad constructora cuyos accionistas mayoritarios son financieras e inmobiliarias.

${ }^{11}$ October 2008 Monthly Energy Review. Energy Information Administration.

${ }^{12}$ Dow Jones \& Company 
ese entonces. La crisis bursátil de 1987 y la crisis japonesa llevan a una nueva etapa de descenso en el crecimiento de la economía mundial hacia 1989. Si bien no existe una caída anual en el producto bruto de los países medido por su poder de compra (PPP), se manifiesta una caída en la tasa de crecimiento comparable a la de principios de los ' 80 . Situación ésta que persiste hasta el año $1993^{13}$. Aún con la leve recuperación en el crecimiento a partir de 1993, no se alcanzan los niveles de crecimiento anteriores a 1988. Y en esto consiste gran parte de la diferencia entre la suba de fines de los '70 y las de los ' 90 . No existía, como en los '70, una ingente necesidad de poner a producir nuevos pozos.

En 1997, con la crisis asiática y la consecuente reducción en el crecimiento del producto bruto mundial ${ }^{14}$, el precio del petróleo cae llegando en 1998 a un precio constante 50\% más bajo que el de 1986, incluso el más bajo desde 1973 .

Las oscilaciones de este período no correspondieron a oscilantes variaciones en el valor de la extracción del petróleo, sino a diferencias entre la oferta y la demanda, las cuales, a su vez, obtuvieron reacciones diversas del precio, al encontrarnos con una relación producción/capacidad de producción, diferente respecto al de una década atrás. De esta forma, acorde con los precios alcanzados hacia mediados de los '80, éstos rondan un promedio que se sostiene desde esos años durante la década de los '90. Este precio oscilante respecto a un promedio relativamente estable para estos años, sumado a niveles de crecimiento que se mantuvieron con una tendencia decreciente desde fines de los ' 80 , implicaron el sostenimiento de una caída en los niveles de inversión. Un indicador que refleja este proceso de desinversión es el presupuesto de los gobiernos de los países miembros de la Agencia Internacional de Energía (IEA) destinados a inversión en combustibles fósiles en general ${ }^{15}$ (CUADRO 6).

\footnotetext{
${ }^{13}$ Datos FMI.

${ }^{14}$ Medido en PPP, datos FMI.

15 Los países miembros de la AIE son: Australia, Austria, Bélgica, Canadá, República Checa, Dinamarca, Finlandia, Francia, Alemania, Grecia, Hungría, Irlanda, Italia, Japón, Corea, Luxemburgo, los Países Bajos, Nueva Zelanda, Noruega, Portugal, España, Suecia, Suiza, Turquía, Reino Unido y los Estados Unidos. Debido a la falta de datos, la República Checa no está incluida en el estimado del total.
} 
Características de la producción petrolera a partir de los años 70:

Un análisis del caso argentino en el esquema de acumulación mundial

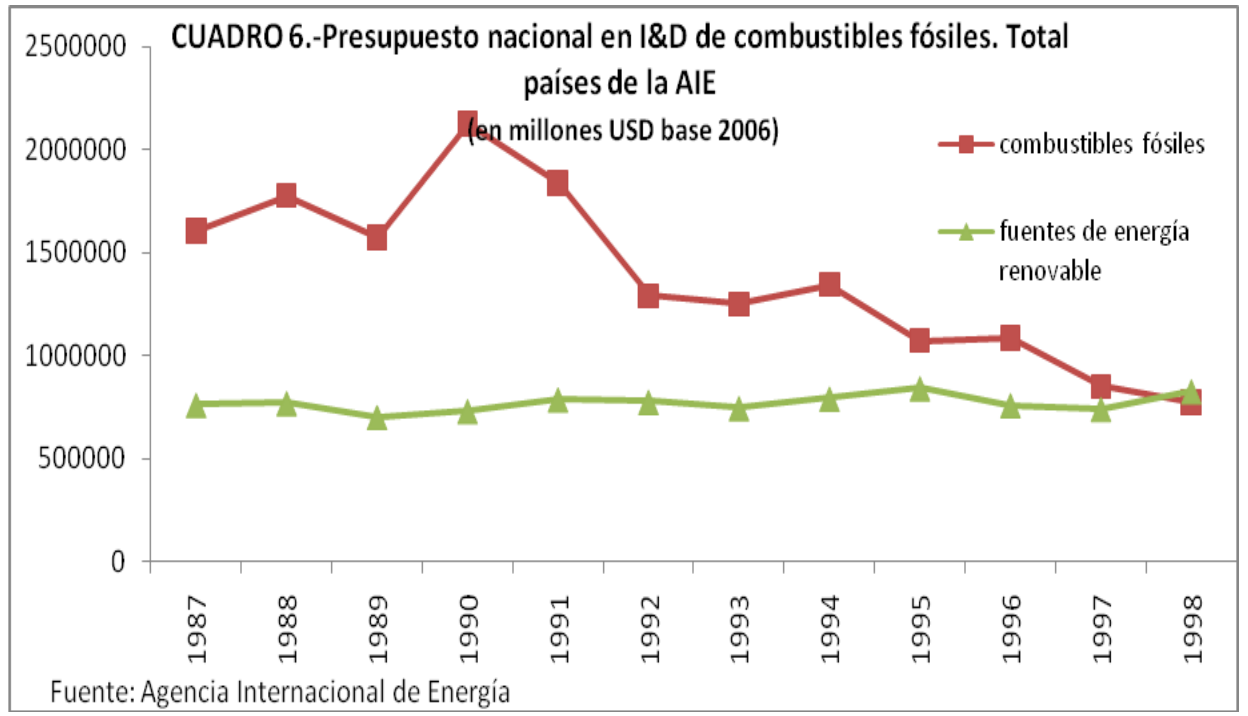

Un informe realizado por Ernst \& Young para el American Petroleum Institute sobre las inversiones privadas en el sector para el período 1992-2006, refleja la caída de "inversión nueva", respecto a las utilidades netas de 57 de las compañías petroleras más grandes a nivel mundial ${ }^{16}$. La "inversión nueva" se refiere a la inversión en propiedades, plantas y equipos, y a los gastos en exploración, investigación y desarrollo. La suba que se observa para el año 1998 responde a una caída de la utilidad neta para ese año a menos del $50 \%$ del monto correspondiente al año anterior como consecuencia de la crisis asiática (CUADRO 7).

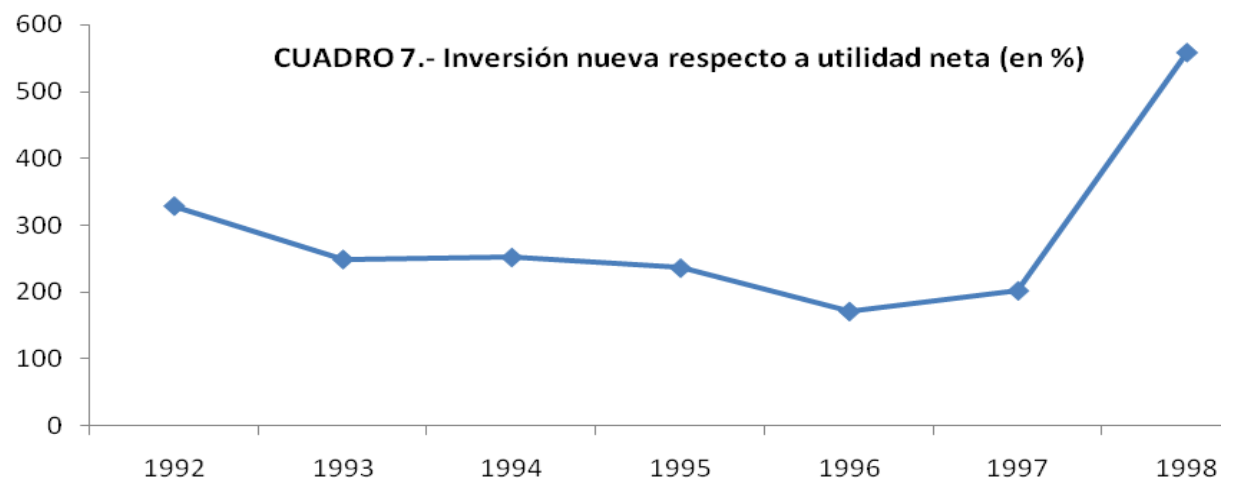

Fuente: en base a datos de informe Ernst \& Young

${ }^{16}$ Investment and other uses of cash flow by the oil industry,1992-2006. Prepared by Ernst\&Young LLP for the American Petroleum Institute, Mayo 2007. 
La caída en los niveles de inversión resultaron, a diferencia del período anterior, en el estancamiento, e incluso leve caída, del ratio reservas/producción (CUADRO 8), fenómeno similar al de la Argentina como se puede observar en los datos anteriormente expuestos en la TABLA 1.

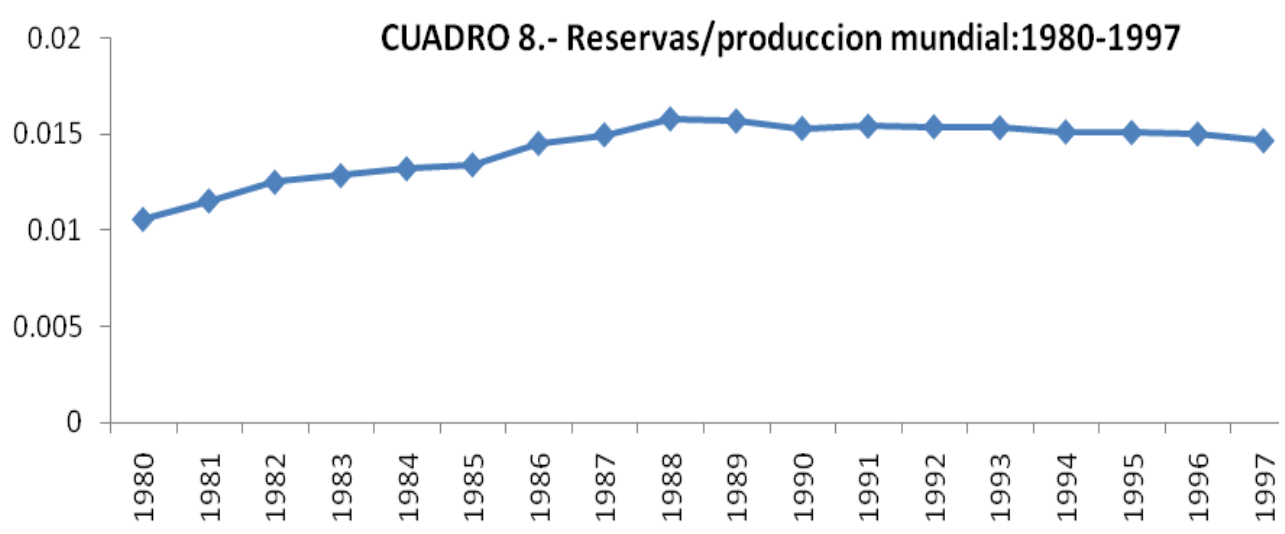

Fuente: BP statistical review

El avance del capital financiero se refuerza durante la década del '90, donde los países subdesarrollados desregulan sus mercados para la entrada masiva de capital, ofreciendo mercados que típicamente formaban parte de la órbita estatal, así como altas tasas de interés, garantías en el tipo de cambio, etcétera. Efectivamente, la privatización de YPF se dio en una primera etapa a través de capitales locales y fondos de pensión norteamericanos y entidades financieras. La búsqueda de ganancias de corto plazo y la producción en vistas a intereses de capitales extranjeros de mayor tamaño que operan a nivel regional, se expresan aquí también en una disminución en las inversiones de riesgo y de largo plazo y en el aumento de la producción a fines de su exportación. En este sentido es que señalamos a las reformas energéticas en Argentina como parte de una transformación en el capital petrolero mundial.

\section{El nuevo milenio y el boom en el precio del petróleo}

Posteriormente, a la crisis asiática, la reducción en la tasa de acumulación y la consecuente deflación llevan a un nuevo proceso de centralización de capitales. Se produce una ola de fusiones y adquisiciones que sacudió a todos los segmentos de la industria petrolera, y que concluye con la existencia de tres grupos petroleros principales hacia el año 2000: Exxon-Mobil, BP-Amoco-Arco y Royal Dutch Shell. 
Por otra parte, en 1999 se redefine un estricto acuerdo sobre las cuotas de producción entre los países de la Opep, que lleva nuevamente a una caída anual de la producción petrolera mundial. Conjuntamente con la posterior recuperación de la demanda asiática, el año 2000 se encuentra con un precio del petróleo en alza, llegando a un $124 \%$ más que dos años antes. Aún así, a precios constantes, recién alcanzaba el pico alcanzado diez años atrás.

A partir del año 2000, con la crisis de las acciones llamadas "tecnológicas", nuevamente se acrecentó el monto de capitales destinados a invertir en mercancías como el petróleo $\mathrm{u}$ otros productos provenientes de la tierra que normalmente acompañan el proceso inflacionario. La tasa de interés real de EEUU cae en el 2001 y se mantiene en porcentajes bajos, e incluso algunos años negativos, durante el período 2001-2006 ${ }^{17}$, con lo cual el rendimiento de bonos u otro tipo de instrumentos no ofrecía grandes expectativas para el capital financiero. Es por ello que para principios del milenio, con la suba de precios de petróleo, puede visualizarse un comportamiento del capital que apuntó al incremento en las reservas probadas mundiales, y que en Argentina se ve también en un aumento en sus reservas a partir del crecimiento en los metros perforados totales para los años 2000 y 2001

Sin embargo, esta situación se revierte hacia el año 2003. Para este año se recuperan las tasas de crecimiento de la economía mundial, crece la producción de petróleo y comienza un ciclo de suba histórica de su precio que durará aproximadamente cinco años. En este lapso de tiempo, el precio del crudo aumenta más de un 150\%, siendo el 2004 y 2005 los años de mayor suba (33\% y 42\% respectivamente). Si se compara el comportamiento del precio con el de la tasa de crecimiento mundial medida por PPP, se podría comprobar un comportamiento similar de ambos indicadores entre 1980 y 2004. Pero la escalada del precio entre el 2004 y 2007 no presenta la misma correlación -lo mismo ocurrió con la suba de precios de los '70-. Esta suba fue similar en el precio de otros productos de la tierra, lo que se relaciona con las determinaciones de su precio, en donde el contexto de una crisis y de bajo rendimiento financiero puede generar una burbuja especulativa sobre la tierra y de esta forma trasladar el alto precio de la misma a un precio de monopolio creciente sobre este tipo de mercancías. El derrumbe del precio se haría efectivo en el curso del desenvolvimiento de la crisis y la recesión que ésta conlleva ${ }^{18}$. La incorporación del capital financiero a la producción petrolera (y agraria) es un factor determinante en la especulación sobre la tierra, el traslado de su alto precio a los precios de los minerales (o cultivos) y, en este sentido, cierto desajuste en la relación precio-inversión.

${ }^{17}$ Federal Reserve Bank of St Louis y U.S. Department of Labour.

${ }^{18}$ La crisis financiera resultó en un hecho en septiembre del 2008 revelándose justamente el mercado hipotecario como uno de los detonantes del proceso deflacionario subsiguiente. En este contexto el precio del petróleo retrocedió a los USD 41 el barril (diciembre 2008) cayendo un $60 \%$ en cuatro meses. 
En vista de estas determinaciones, existe un fenómeno que se contrapone al perseverante aumento en la producción mundial de petróleo-que se muestra indeclinable entre el 2003 y el 2006-, en la del precio y en la de la masa de capitales puestos a producir en el sector; se trata de la magnitud mundial de reservas probadas. Si bien éstas aumentan en volumen, el ratio reservas probadas/producción comienza a descender a partir del 2002, mostrando una leve recuperación hacia el 2006 (CUADRO 9). El aumento observado para los años 2001 y 2002 corresponde a la caída en la producción mundial de crudo.

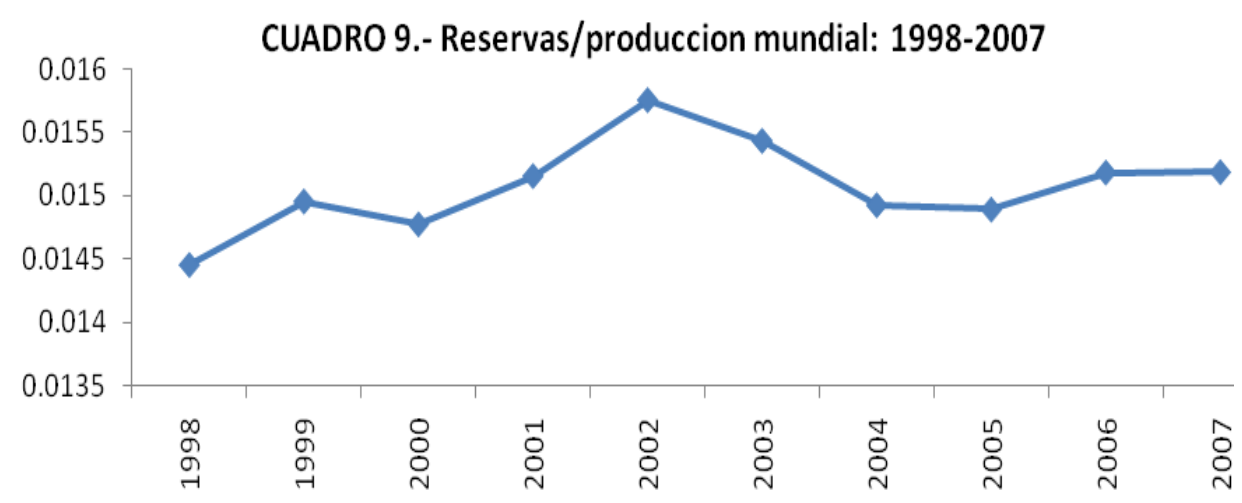

Fuente: BP statistical review

La suba del precio en el nuevo milenio no se resolvió en un aumento en las reservas/producción como la que ocurriera a partir de 1980, en que aumenta en ocho años en casi un $50 \%$.

En Argentina, hacia 1999, la producción de petróleo local comienza a caer, lo cual se condice en una primera etapa con las tasas de crecimiento real negativas para el período 1999-2002 (ver CUADRO 5). Teniendo en cuenta que las exportaciones se habían convertido en eje de la producción local, justamente para este mismo año se sucede la crisis brasilera y la devaluación del real, por lo que las exportaciones caen en un 18.6\%. Para el año 2000, se recuperan las exportaciones, aumenta el precio internacional del petróleo, y aumenta la cantidad de metros perforados totales, aunque caen los correspondientes a exploración (CUADROS 10 y 11). Como se mencionara anteriormente el aumento en los metros perforados totales también continúa para el año 2001 en que crecen las reservas a nivel mundial. Por otra parte, era inminente la crisis de convertibilidad en Argentina, por lo que el aumento de la producción se realizaba mediante el endeudamiento de las empresas que, a su vez, remitían al extranjero las divisas obtenidas, actuando como verdaderos agentes financieros ${ }^{19}$.

${ }^{19}$ Id. Kozujl (marzo 2005) 

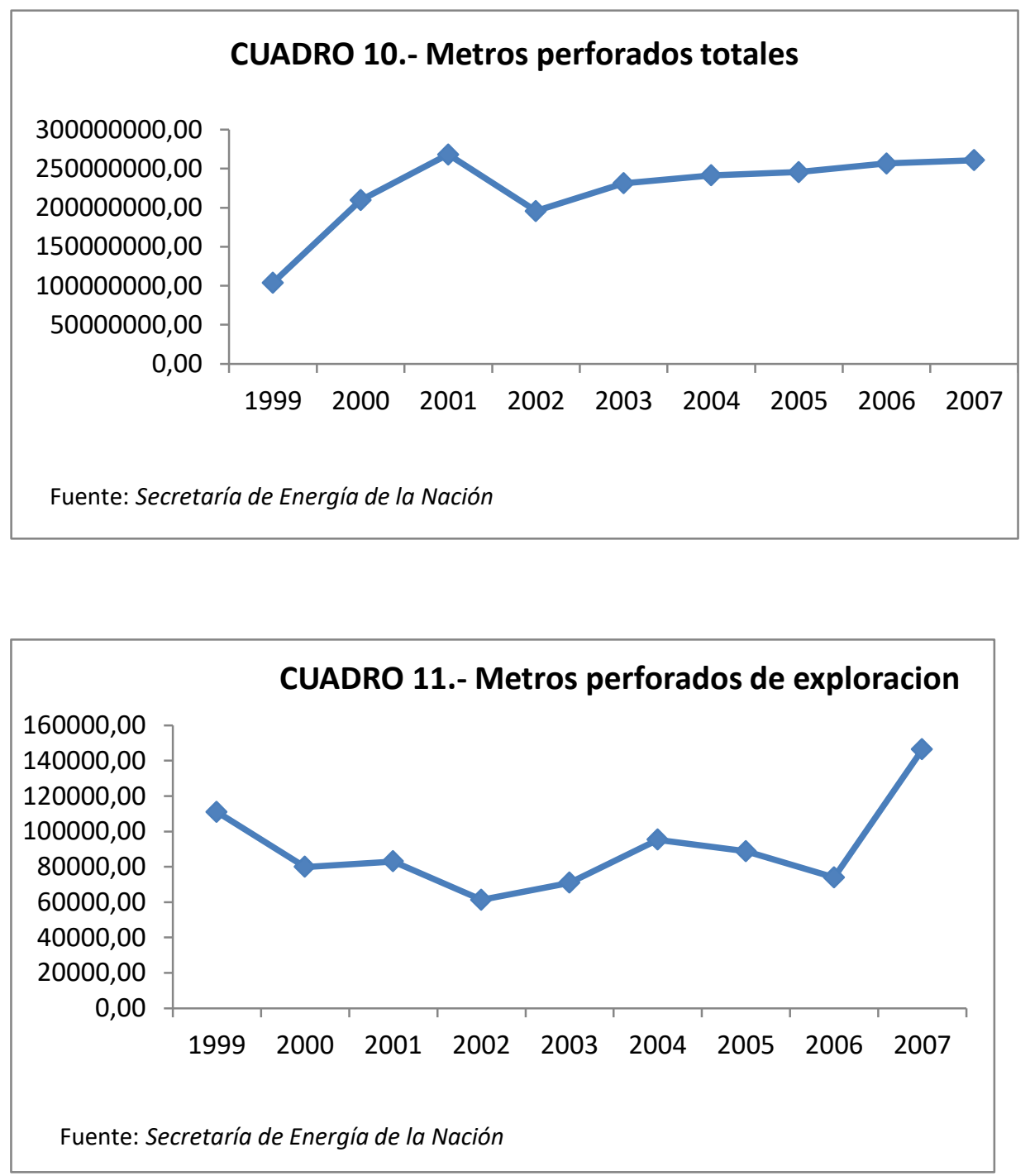

La caída en las tasas de crecimiento para principios del milenio también se manifestó en Argentina. En el 2001, se desata una crisis que lleva al siguiente año a la devaluación del peso. Hacia 2002 se dan los niveles históricos más bajos en metros perforados de exploración. Es el año en que cae el precio internacional de crudo, y en donde se reorientan las inversiones energéticas hacia otros países de América Latina (Bolivia, Brasil).

La caída de precios local e internacional se revierte hacia el año 2003, pero desde este año los precios locales quedan más rezagados (ver CUADRO 1). Ciertos acuerdos los van ajustando, pero la crisis del 2002 y la devaluación conllevaron a un mayor control de los precios. Básicamente era insostenible con la devaluación el sostenimiento de los precios en dólares para el consumo interno. Pero, con la 
subida de precios en el 2003, los metros perforados totales se recuperan, aunque en el 2005 los de exploración vuelven a caer, y suben en el 2007, lo que podría explicarse a partir del proceso de especulación ya mencionado sobre los productos de la tierra frente a una inminente crisis financiera. Aún así continúa la tendencia decreciente en la producción local, lo que expresa por su parte una productividad media menor por pozo especialmente a partir del 2003, como efecto del progresivo agotamiento de las reservas existentes. La causa está en la ausencia de reservas incorporadas en áreas nuevas o de reciente descubrimiento ${ }^{20}$.

Como consecuencia del vaciamiento de las reservas probadas de petróleo, la relación reservas/producción argentina que venía creciendo desde 1995, con el aumento en la tasa de extracción, a partir del 2001 se estanca e, incluso, cae para el año 2006 (CUADRO 12). El aumento de reservas no se asoció al aumento de perforación de pozos exploratorios, lo que implica que incluso a partir del 2001 las reservas absolutas caigan. Asimismo, el ciclo de suba del precio que se inicia en el 2003 no se tradujo en la recomposición de las reservas.

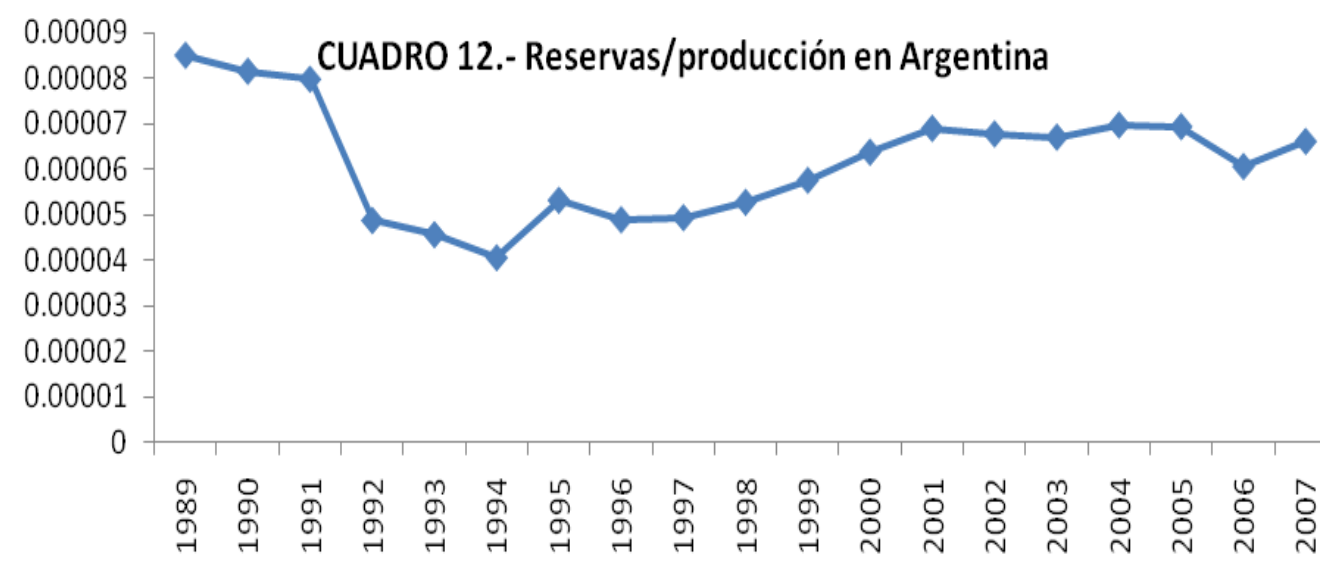

Fuente: elaboración propia en base a datos de Secretaría de Energía de la Nación y Energy Information Administration

\section{Algunas determinaciones de la inversión petrolera}

El progresivo agotamiento del mineral, lleva a que el precio del petróleo se encuentre cada vez más tensamente ligado al aumento de su extracción por un lado, y al aumento de la productividad del trabajo en la producción petrolera por el otro. El aumento en su extracción estará determinado por la demanda, o sea por la acumulación de capital total, y de ella dependerá la mayor productividad del trabajo en general. En cuanto a la productividad del trabajo en la producción de

${ }^{20}$ Id. Kozujl (julio 2002) 
petróleo, el aumento en las dificultades de su extracción implica que el capital incorporado tiene una productividad decreciente, lo cual se expresa en la caída de la productividad media de los pozos. Esta es una de las determinaciones de la creciente relación entre el crecimiento económico y el precio del petróleo, a partir de la década de los ' 80 , lo cual de forma opuesta se compensa con el aumento en la participación de energías alternativas y la eficiencia energética. Esta limitación es principalmente superada con el desarrollo tecnológico que incremente la productividad de los pozos (como por ejemplo las innovaciones en recuperación secundaria o terciaria), o que permita la incorporación de nuevos yacimientos que se revelen como productivos frente a las nuevas condiciones de producción. La diferente velocidad en que la productividad del trabajo concreto que afecta de manera directa la producción petrolera y el trabajo medio general se diferencien entre sí, se expresa en la volatilidad del precio. La volatilidad en parte se potencia por la mayor manipulación en la oferta disponible de crudo, ya sea por la posibilidad del retiro de la producción o por la acumulación de existencias, que se agudiza con el agotamiento del mineral y la centralización de su producción.

De esta manera, la incierta capacidad de oferta futura, de valor y de precio, que de conjunto son indicadores que prometen una tasa de ganancia para el capital, obstruye la fluidez de su movimiento hacia esta esfera productiva, especialmente en la medida en que la masa de capital necesario para el aumento de la productividad del trabajo es cada vez mayor. A su vez, la creciente dificultad de extracción de ciertos minerales, da lugar a un ciclo de valorización del capital más largo e, inclusive, más riesgoso que en otro tipo de actividades. Para el caso del petróleo, el ciclo de valorización a partir de la etapa de exploración es de alrededor de 5 años o más. En este sentido, estas barreras a la incorporación de capital a la producción se convierten en otra determinación de la mayor volatilidad en el precio.

Las necesidades de capitales cada vez mayores y las crecientes dificultades en su valorización son determinantes del ingreso de capitales financieros a la producción de este tipo de mercancías, así como también de la intervención del estado en tanto se trata de un recurso esencial para el capital total. La perspectiva de una crisis financiera acrecentada a partir del 2004, dificultó aún más esta cuestión, aunque dio lugar a un aumento del precio por la especulación de la tierra. Pero en tanto esta fuera la causa de la abrupta suba del precio -especialmente a partir del 2004-, la consiguiente recesión no permitirá una recuperación del capital invertido, lo que explicaría que la suba del precio de los últimos años se habría tomado sólo de forma circunstancial, como señal de inversión de nuevo capital.

Es en el marco de este desarrollo, en que puede comprenderse la fuerte suba del precio en el nuevo milenio, sin un consecuente aumento de las reservas probadas ni en la Argentina ni en el mundo, como sí sucediera posteriormente a la suba de fines de los '70. Las Reservas Recuperables Estimadas (EUR), se calcula que han sido consumidas en un $67 \%$ en Norteamérica, superando el punto medio de explotación. En el Medio Oriente este cálculo es del 24\%, mientras que en el mundo de un $37 \%$, con lo cual se calcula el peak oil mundial -al nivel de crecimiento de producción actual y dada la tecnología existente- para el año 2030, punto a 
partir del cual el valor del petróleo comenzaría a aumentar considerablemente ${ }^{21}$. La caída en la expectativa de crecimiento por las regulares crisis del capital llevó en el nuevo milenio a reforzar la desinversión en el sector. El crecimiento de reservas de estos años que se diera en ciertas regiones (básicamente en los países de la Opep), proviene, especialmente a partir del 2004, de yacimientos existentes y no del descubrimiento de nuevos pozos 22 . Por otra parte, un informe de la FSA, agencia reguladora del mercado de futuros británico, afirma que frente a la suba del precio del 2003 los productores petroleros no aumentaron su capacidad productiva, sino que invirtieron mayormente en acciones de otras empresas ${ }^{23}$. Ya en el año 2000, en la cumbre II de Caracas, la OPEP demostró su inquietud en realizar un fondo de impacto para la prevención de la caída de la renta por la eficiencia tecnológica o el avance en la sustitución del petróleo. En el 2007 la OPEP remarcó un escenario de caída de crecimiento de la demanda hacia el 2030, de entre el 3\% y el $13 \%$, argumentando así su reticencia a una fuerte expansión en la inversión, a fines de evitar una caída de los precios que perjudique a los países miembros ${ }^{24}$.

\section{Centralización y estado en esta nueva etapa}

Los primeros pasos hacia la centralización en los comienzos de la industria petrolera, apuntaban a la integración entre las diferentes etapas de la producción que dieran un mayor control y organización del proceso productivo. Es decir, era un factor de potencia para la productividad del trabajo invertido en la producción del petróleo. Posteriormente, la creciente acumulación del capital impulsó cada vez más la centralización de capitales especialmente en períodos de guerra y recesión.

Con el crecimiento de esta industria, su desarrollo tecnológico y la propia concentración de los capitales individuales, surgieron nuevos productores que lograron hacerse de un lugar en un mercado en amplio crecimiento. Aún así, a partir de las características propias de este tipo de producción, el capital mínimo necesario para producir fue creciendo distinguidamente con el progresivo agotamiento de los pozos petroleros, ya sea por la necesidad de superar la productividad decreciente del capital como para explorar nuevos yacimientos. Esta característica cobró relevancia frente a la necesidad del capital social de diversificar las fuentes de suministro por el conflicto de intereses con los estados del Medio Oriente. Efectivamente, las particularidades en la renta minera implican un mayor poder de manipulación de la oferta por parte de los dueños de los pozos, lo que eventualmente modifica de forma abrupta la estructura de renta diferencial

${ }^{21}$ World Energy Council, Survey of Energy Resources 2007.

22 id. World Energy Council

${ }^{23}$ FSA Markets Infraestructure Department, Growth in Commodity Investment, marzo 2007.

${ }^{24}$ OPEC, World Oil Outlook 2007. 
mundial. De esta manera, y sumado a la centralización de hecho de los pozos, en tanto el agotamiento del mineral lleva al cierre de aquellos más pequeños, el capital necesita de un mayor nivel de concentración para quebrar con este progresivo aumento en el monopolio del suelo.

Ya pasada la recesión y deflación de los '80, y con un progresivo retorno a los pozos más productivos del Medio Oriente, podría decirse que el nivel de centralización de capital del sector alcanzó un grado por encima del existente en las industrias en general. Aún así, la cuestión que surge es si la progresiva desaparición de la competencia puede desembocar en una nueva traba al aumento de la productividad del trabajo en esta actividad y, consecuentemente, a una deficiencia en oferta energética. La necesidad de incorporar nueva tecnología en la producción, es el eje de este cuestionamiento, y tiene dos claras perspectivas: la del aumento en el rendimiento de los pozos existentes, o la del aumento en exploración para la incorporación de otros nuevos. La primera veta es más accesible para los niveles de concentración actual, pero tienen un límite físico insuperable, la segunda requiere de inversiones más cuantiosas y se focalizan en la explotación offshore. Existe también una tercera posibilidad que es la inversión en energías alternativas; en caso de que existiera la posibilidad de que, en la actualidad, éstas suplanten el nivel de demanda energética existente, las convertiría en el insumo energético más caro para el capital, pero esto, nuevamente, dadas las condiciones tecnológicas actuales.

En definitiva, el cuestionamiento se basa en que el capital petrolero no disponga de la inversión necesaria para las necesidades del capital conjunto de la sociedad que requiere del abastecimiento constante de energía, y necesariamente, en términos de un valor cada vez menor. Por otra parte, este límite podría devenir en una segunda problemática, que son las necesidades de abastecimiento de aquellos capitales nacionales menores a los dispuestos para la producción de energía y que, muchas veces, se encuentran representados por estados poseedores de yacimientos de petróleo. Aquí y, a diferencia de lo cuestionado anteriormente, la competencia de capitales en general se transforma en un factor de conflicto en torno a este recurso.

Estas situaciones se manifestarían en un nuevo -o más bien crecientefactor de volatilidad del precio y de su suba frente al aumento de su demanda, lo que perjudica claramente al capital conjunto, consumidor esencial del hidrocarburo. Es relevante considerar aquí un tercer cuestionamiento, que es la capacidad de producción energética respecto a una necesidad social que no se exprese como necesidad del capital, y que implique un deterioro de las condiciones de vida de un sector de la población. Si bien esto generaría un debate sobre la posibilidad o no de abstraer las necesidades respecto del modo de producción general. En este sentido, es menester señalar el efecto material sobre la humanidad que esta forma específica de producción tiene en cuanto a la asignación de su producto.

En tanto, el aumento en la productividad de la producción de energía es una necesidad del capital total, frente a la progresiva desinversión -por lo menos la que requiere de la superación física del agotamiento del mineral en un mediano y largo plazo-, y como forma de socializar el riesgo entre el conjunto de los capitales, 
existiría una nueva determinación de que el estado ocupe el papel principal como productor o el fortalecimiento de su papel regulador sobre aquellos recursos que expresen su escasez relativa a las necesidades del capital. La alta concentración geográfica de los yacimientos petroleros, convierte a la extracción de petróleo en una actividad de características monopólicas. Esto es una determinación de la potencial intervención del estado en la obtención de este recurso básico en la producción general de mercancías. Por ello, en general los principales terratenientes de los yacimientos minerales son el propio estado, ya sea que los explote de manera directa o los otorgue en forma de concesión, y es la razón por la cual el capital requirió de leyes que otorguen los recursos naturales a la propiedad colectiva, garantizando así su acceso a los mismos. Pero estos estados, como se mencionara anteriormente, bien pueden ser diferentes a aquellos que representan el interés del capital concentrado dispuesto a producir el mineral en cuestión, con lo cual si bien son dependientes el uno del otro. La cuestión del precio y del suministro de la mercancía puede resultar en conflictos políticos o incluso bélicos, que expresan las diferentes necesidades de los capitales individuales representados bajo la órbita de un estado-nación. En este sentido, los límites de los capitales individuales frente a las inversiones mínimas requeridas no sólo determinan la mayor incorporación del estado a la producción, sino la confrontación de los estados entre sí.

\section{Conclusiones y Perspectivas}

El comportamiento de las inversiones en producción de petróleo, tanto en Argentina como en el mundo, evidencia una transformación en el sector, por la cual el aumento en la producción e incluso en los precios no se expresa en un desarrollo tecnológico significativo que apunte a la incorporación de nuevos yacimientos. La expectativa en la tasa de ganancia frente a capitales mínimos cada vez mayores en concentración y en riesgo, implica que el precio, que normalmente es la señal para la inversión de capital en una rama productiva, se desligue más de su función como determinante en la asignación de trabajo social necesario para la reproducción de esta mercancía.

El avance del capital financiero en el sector permitió, en primer lugar, la disposición de los capitales necesarios para invertir durante los '70, y luego la centralización de la producción necesaria ya desenvuelta la crisis por la sobreproducción de los años anteriores. Asimismo condicionó la organización de la producción al interior de las unidades productivas y las características de las inversiones, en donde aquellas de corto plazo y menor riesgo comenzaron a ganar protagonismo. Esta transformación se evidenció con sus características específicas en Argentina, donde la privatización de YPF conformó un nicho de ganancias para el capital, especialmente para aquel cada vez más desligado de los procesos productivos, en el marco mundial de absorción de empresas infravaloradas por desinversión y endeudamiento. 
Sin embargo, el progresivo agotamiento de las reservas existentes implica una contradicción para el capital de conjunto. La recesión que recién comienza apuntará a una reducción en la extracción de petróleo y, en consecuencia, a una necesidad de mayor centralización en la producción. Pero en tanto los capitales privados continúen ejerciendo el grueso de la producción, y la desinversión de largo plazo y de riesgo sea la característica histórica frente al avance del capital financiero, una posterior recuperación de la economía llevará al aumento del precio del petróleo nuevamente. La tolerancia en la caída de la tasa de ganancia de los capitales consumidores de crudo, o sea, del capital conjunto de la sociedad, determinará la necesidad de una mayor incorporación del estado en la producción a fines de salvaguardar sus intereses. Pero, a diferencia de otros momentos históricos, nos encontramos en un período no sólo de crisis financiera, sino de estados quebrados cada vez más imposibilitados de resolver las contradicciones propias del modo de producción que representan. Las sociedades mixtas entre los capitales privados altamente concentrados junto a organizaciones o compañías estatales, pareciera ser la perspectiva en la forma de organización de la producción petrolera frente a la necesidad de inversiones de más largo plazo y riesgo para incorporar nuevos yacimientos y desarrollar fuentes de energía alternativas.

En este contexto, la apropiación de la renta petrolera es un factor más de competencia entre los capitales, así como también lo es su competencia en tanto consumidores de petróleo. De esta manera, los intereses de los capitales nacionales que no disponen de un nivel de concentración necesario para satisfacer progresivamente su demanda energética, caso que se correspondería con la situación actual en la Argentina, encuentran en la forma actual de inversión y producción de petróleo una contradicción creciente que se revelará de forma acuciante frente a una posible recuperación. Esta determinación implicará aquí un mayor rol del estado nacional, frente a la producción energética local. Existen diversos mecanismos para realizarlo, en donde encontrados intereses intentarán satisfacer la necesidad de acumulación tanto del capital local como del extranjero que opera en el país. De aquí que existan diferentes recursos a los que el estado pueda acudir como representante de estos intereses. Por un lado, se encuentra la reestatización de YPF o la nacionalización parcial transformándola en una sociedad mixta con mayor participación estatal en la producción. Sin embargo, en la medida en que los estados nacionales tampoco puedan afrontar las inversiones necesarias y en tanto sean cada vez más dependientes de los capitales mundiales altamente concentrados, acudirán ya sea a un mayor endeudamiento, y/o al avance en la integración regional con capitales y estados de la región que estén más estrechamente vinculados al mercado local. Esto, a su vez, podría conformar una capacidad de formación de ganancia mayor para los capitales mundiales de gran concentración que, por tanto, se vean interesados de incorporarse a esta integración energética regional. Aquí se ve cómo la centralización de los capitales privados y el interés de los mismos se consuman mediante la gestión y recursos de los estados y la socialización de los riesgos entre todos los capitales individuales. 
El límite de acción de los actuales estados en tanto actores de centralización productiva se encuentra, a su vez, en la contradicción de ser una manifestación de la forma de organización actual basada en la propiedad privada. Por otro lado, será cada vez más evidente esta determinación a través de la creciente contradicción entre las necesidades del capital y las sociales en general, lo cual se expresa en la razón de ser de las crisis mismas. Es decir, en tanto la quiebra de fuerzas productivas es el factor de rescate del capital, ello resulta en la creciente porción de necesidades humanas insolventes sobre la base de una mayor capacidad productiva. De esta forma, la problemática energética debe ser considerada en el marco de las necesidades a las que hace referencia, en términos de caracterizar el problema y por lo tanto su resolución.

\section{Bibliografía}

Doyle E., Hill J., Jack I., Growth in Commodity Investment: risks and challenges for commodity market participants, FSA Markets Infraestructure Department, Marzo 2007, disponible en www.fsa.gov.uk

Energy Information Administration, October 2008 Monthly Energy Review, disponible en www.eia.doe.gov

Ernst\&Young LLP for the American Petroleum Institute, Investment and other uses of cash flow by the oil industry,1992-2006, Mayo 2007, disponible en www.api.org

Gadano N., Determinantes de la inversión en el sector petróleo y gas de la Argentina, de Recursos Naturales e Infraestructura, CEPAL, Santiago de Chile, Octubre 1998.

Kozujl R., Balance de la privatización de la industria petrolera en Argentina y su impacto sobre las inversiones y la competencia en los mercados minoristas de combustibles, División de Recursos Naturales e Infraestructura, CEPAL, Santiago de Chile, Julio 2002.

Kozujl R., Crisis de la industria del gas natural en la Argentina, División de Recursos Naturales e Infraestructura, CEPAL, Santiago de Chile, Marzo 2005.

OPEC, World Oil Outlook 2007, disponible en www.opec.orgPistonesi, Figueroa de la Vega y Torres, Política de precios de la energía en la Argentina 1970-1989, Instituto de Economía Energética, Fundación Bariloche 1990.

Rabinovich G.,Riavitz R., Cattaneo F., Análisis del mercado de combustibles líquidos en la República Argentina. Comportamiento de las naftas y el gasoil desde la desregulación petrolera (1992/1999)", Instituto Argentino de la Energía "General Mosconi", Abril 2000, disponible en www.iae.org.ar

Latitude, vol. 3, n², pp. 41-64, 2009. 
Características de la producción petrolera a partir de los años 70:

Un análisis del caso argentino en el esquema de acumulación mundial

World Energy Council, Survey of Energy Resources 2007, disponible en www.worldenergy.org

Yergin D., La historia del petróleo, Ed. Vergara, Buenos Aires 1992.

Webs consultadas:

- British Petroleum www.bp.com

- Dow Jones Company www.dj.com

- Energy Information Administration www.eia.doe.gov

- Finantial Services Authority www.fsa.gov.uk

- International Energy Agency www.iea.org

- International Monetary Fund www.imf.org

- Organization of the Petroleum Exporting Countries www.opec.org

- Secretaría de Energía de la Nación www.energia.gov.ar

- World Energy Council www.worldenergy.org 